HUBERTUS BUCHSTEIN

\title{
,Gretchenfrage‘ ohne klare Antwort - Ernst Fraenkels politikwissenschaftliche Gemeinwohlkonzeption
}

\section{Einleitung}

Vor gut einem Jahrzehnt bemängelte Fritz W. Scharpf, daß die „Politikwissenschaft vor den Aporien des Gemeinwohlbegriffs weitgehend kapituliert [hat] und damit auf die Weiterführung ihrer eigenen ordnungstheoretischen Tradition faktisch verzichtet" ${ }^{1}$ Scharpf sah in diesem Verzicht einen zentralen Grund für die aus seiner Sicht beunruhigende Landnahme von Rational-Choice-Theorien. Für diese Beunruhigung gibt es heute weniger Anlaß als vor zehn Jahren. Rational-Choice befindet sich in der gegenwärtigen politikwissenschaftlichen Theorielandschaft eher wieder auf dem Rückzug und gleichzeitig wird von verschiedenen Seiten eine Renaissance des Gemeinwohlbegriffs oberhalb der Ebene der Kollektivgutproduktion rationaler Akteure konstatiert. Bemerkenswert ist freilich, daß diese Renaissance zu keiner eindeutigen Begriffsverwendung von ,Gemeinwohl' in der Politikwissenschaft geführt hat. Im Gegenteil. Dem Leser einschlägiger neuer Publikationen zum Thema drängt sich der Eindruck einer positiven Korrelation zwischen der Häufigkeit des Rekurses auf den Gemeinwohlbegriff und der Vielfältigkeit seiner Bedeutungen und Verwendungsweisen auf. Diese Unklarheiten liegen zudem im fundamentalen Bereich der Begriffsdefinition. So herrscht keine Einigkeit darüber, wer von dem ,Gemein " umfaßt werden soll und wer als Ausgeschlossen zu gelten hat (in Luhmanns Terminologie: die soziale Dimension), auch nicht darüber, was unter deren ,Wohl' zu verstehen ist und wie es festzustellen ist (die sachliche Dimension), auch nicht über den darin jeweils anvisierten Zeithorizont (die temporale Dimension) und schließlich auch nicht über die Frage, wie ein auch immer geartetes Gemeinwohl politisch zu realisieren ist (die praktische Dimension). ${ }^{2}$

Grundsätzlich konkurrieren in den klassischen wie modernen Gemeinwohlvorstellungen drei Grundmodelle, ein objektivistisches, ein Schnittmengenmodell und ein deliberatives Modell. Sie lassen sich stichwortartig folgendermaßen charakterisieren:

1 Scharpf 1991, S. 624.

2 Diese und andere Unklarheiten der „sozialen Referenz" (Offe) des Gemeinwohlbegriffs werden gut aufgelistet in Offe 2002. 
(a) Das objektivistische Modell. In der Vorstellung eines ,objektiven' Gemeinwohls soll das Gemeinwohl sowohl erkennbar sein als auch als Orientierungspunkt praktischer Politik fungieren können.

(b) Das Schnittmengenmodell. Eine zweite Vorstellung besteht in der Interessenallgemeinheit des generalisierten Egoismus; in diesem Modell besteht das Gemeinwohl aus der Schnittmenge aller empirisch vorfindlichen Interessenlagen. Die institutionelle Konsequenz dieses Modells besteht in einer möglichst breit gestreuten Veto-Positionierung politischer Akteure. Nur solche Inhalte verdienen das Gütesiegel ,Gemeinwohl', die von allen Angehörigen eines politischen Systems geteilt werden. Folgt man Robert E. Goodin, so stand diese Vorstellung des Gemeinwohls Pate beim Plädoyer der amerikanischen Autoren der Federalist-Papers für ihr weitgehendes Gewaltenteilungsmodell. ${ }^{3}$ Wenn die interne Differenzierung und Pluralisierung der Überzeugungen und Interessen der Mitglieder eines politischen Gemeinwesens zunimmt, dann wird die Schnittmenge entsprechend kleiner oder verschwindet gar ganz. Politik, die auf das ,Gemeinwohl' zielt, muß so an Stellenwert verlieren. Diese Überlegung deckt sich mit der Kritik einer großen Zahl heutiger Beobachter des amerikanischen Regierungssystems, nach denen das dortige Modell für die mangelhafte Bearbeitung von, public interests' in der gegenwärtigen amerikanischen Politik verantwortlich ist.

(c) Das deliberative Modell. Eine dritte Vorstellung von Gemeinwohl zielt auf eine kommunikative Allgemeinheit. Danach geht die Genese des Gemeinwohls zwar von gegebenen Interessen und Präferenzen aus, speist diese aber in einen Prozeß der argumentativen Auseinandersetzung ein, so daß am Ende ein Ergebnis stehen kann, das für alle Betroffenen gleichermaßen akzeptabel ist.

Wenn auf der einen Seite begrifflicher Konkretisierungs- und Nachbesserungsbedarf besteht und auf der anderen Seite fundamental differierende Modelle miteinander konkurrieren, dann schlägt zumeist die Stunde der ideengeschichtlichen Vergewisserung. $\mathrm{Zu}$ den Autoren, auf die wir bei einer solchen Vergewisserung der politikwissenschaftlichen Tradition sowohl in neueren Arbeiten zum Thema Gemeinwohl ${ }^{4}$ als auch in sämtlichen einschlägigen Handbüchern und Lexika unter dem entsprechenden Stichwort stoßen, zählt an besonders prominenter Stelle Ernst Fraenkel (1898-1975). ${ }^{5}$ Fraenkel, der von 1951-1970 in Berlin Politikwissenschaft lehrte, nahm explizit für sich in Anspruch, eine eigenständige politikwissenschaftliche Gemeinwohlkonzeption entwickelt zu haben. Er konzipierte sie im Rahmen seiner in den 1950er und 1960er Jahren ausformulierten neopluralistischen Demokratietheorie.

3 Vgl. Goodin 1996.

4 Auch Münkler/Fischer zufolge ist Fraenkels Gemeinwohlkonzeption von besonderer Bedeutung, denn es „betont die gesellschaftlich-kommunikative Schlüsselrolle des Gemeinwohls als auch heute noch unverzichtbaren politischen Begriff" (Münkler/Fischer 1999, S. 250).

5 Ernst Fraenkel zählt mittlerweile zu einem Klassiker der bundesdeutschen Politikwissenschaft. Verschiedene Aspekte seines Werkes sind in der Sekundärliteratur gut aufgearbeitet. Zu nennen sind insbesondere zwei Arbeiten aus dem Hamburger Umfeld von Winfried Steffani, Peter Massings Buch Interesse und Konsens (Massing 1979) und Joachim Detjens voluminöse Studie Neopluralismus und Naturrecht (Detjen 1988). Einen Überblick über die verschiedenen Phasen in Fraenkels Entwicklung geben die Beiträge in dem Sammelband (Buchstein/Göhler 2000). 
Wie sehr Fraenkel die Frage des Gemeinwohls umtrieb, wird schon an der Art und Weise erkennbar, in der er das Thema aufgriff. Fraenkel war von seinem ganzen Duktus her ein ausgesprochener Bildungsbürger, und wenn er bestimmten Thesen und Aussagen ein besonderes Gewicht verleihen wollte, griff er in die Schatztruhe klassischer Zitate und Anspielungen. Spätestens wenn Goethe oder Shakespeare ins Spiel kamen, wußte der geübte Fraenkel-Zuhörer, daß nun die höchste Stufe der Aufmerksamkeit geboten war. Beim Thema ,Gemeinwohl' führte Fraenkel gar beide ins Feld, um die Wichtigkeit des Themas zu unterstreichen. Vielzitiert in der zahlreichen Sekundärliteratur ist folgende Sentenz von ihm: „Eine Politikwissenschaft, die zu dem Phänomen ,Gemeinwohl' nichts zu sagen hat, ähnelt einer Vorführung des Hamlet ohne den Prinzen von Dänemark“. ${ }^{6}$ Ein fast ebenso häufig zitiertes Diktum Fraenkels lautet: „Eine Politikwissenschaft, die der Erörterung der Gretchenfrage aus dem Wege gehen wollte, wie die Verwirklichung eines einheitlichen Gemeinwohls in einer differenzierten Gesellschaft ermöglicht werden kann, würde sich bankrott erklären““ ${ }^{7}$ Wie weiland Doktor Faust, so ist also auch Fraenkel bei seiner Antwort auf die Gretchenfrage um Vollmundigkeit nicht verlegen: „Eine Politologie“, so postuliert er, „die sich nicht um die Klärung des Begriffs, Gemeinwohl' bemüht, verdient den Vorwurf, eine Politikwissenschaft ohne Politik zu sein". 8

Es ist die These dieses Beitrages, daß Fraenkels Vollmundigkeit in einem Kontrast zum sachlichen Überzeugungsgrad seiner Gemeinwohlkonzeption steht. Ich möchte die Plausibilität seiner Gemeinwohlkonzeption im folgenden einer genaueren Prüfung unterziehen. Dazu werde ich zunächst den theoriestrategischen Thematisierungskontext der Gemeinwohlfrage bei Fraenkel kurz in Erinnerung rufen (2). Vor diesem Hintergrund werde ich dann zwei Modi der Kritik Fraenkels an der Gemeinwohlkonzeption von Rousseau unterscheiden. Die eine Form der Kritik erfolgt aus materialer, die andere aus prozeduraler Perspektive. Erst beide Aspekte zusammen ergeben das Gesamtbild der Fraenkelschen Gemeinwohlkonzeption (3). Im nächsten Schritt möchte ich dann die Stellung des Gemeinwohls in Fraenkels normativen Bild des politischen Willensbildungsprozesses in der pluralistischen Demokratie lokalisieren (4). Diese Rekonstruktion leitet zu der Folgefrage, wie Fraenkel den materialen Aspekt seiner Gemeinwohllehre begründet. Fraenkel vertritt nachdrücklich ein naturrechtliches Begründungsmuster, das aber - so mein Einwand - angesichts seiner zentralen Bedeutung für die Gemeinwohlkonzeption frappierend unbestimmt und unbegründet bleibt (5). Im letzten Schritt möchte ich diese Kritik beiseite legen und insofern konstruktiv enden, als auf potentielle aktuelle Aspekte der Fraenkelschen Konzeption hingewiesen wird (6).

6 Fraenkel 1966b, S. 272.

7 Fraenkel 1963b, S. 344; oder auch: „Die Gretchenfrage der dem modernen Industriezeitalter adäquaten Staatsform der parlamentarischen Demokratie lautet, ob es angängig ist, trotz des heterogenen Charakters ihrer Gesellschaftsstruktur von einem Gemeinwohl zu sprechen" (1966b, S. 272).

8 Fraenkel 1963b, S. 339. 


\section{Zum Thematisierungskontext der Gemeinwohlfrage bei Fraenkel}

Angesichts der zitierten zentralen Bedeutung des Gemeinwohlbegriffs für Fraenkel ist es einigermaßen befremdlich, wie unsystematisch er den Terminus in seinem Schriften verwendet hat. Bemerkenswert ist zudem, daß der Begriff erst ab 1960 im Zuge der Ausformulierung seiner neopluralistischen Demokratietheorie häufiger in seinen Arbeiten auftaucht. Nicht vom Wortlaut, aber von der Sache her, um deren Willen er die Kategorie des Gemeinwohls bemüht, hat sich Fraenkel zeitlebens der Frage von Normen im staatsrechtlichen und politischen Urteil befaßt. Für Fraenkels gesamtes späteres Werk gilt, daß die Kapitulation der Weimarer Republik und die unrühmliche Rolle, die die deutsche Staatsrechtslehre dabei spielte, prägend war.

Fraenkels Überlegungen zum Thema Gemeinwohl blieben zeitlebens geprägt von seinen ganz persönlichen politischen Erfahrungen. In der Weimarer Republik überwog die Ablehnung des Gemeinwohlbegriffs, etwa wenn es in ideologiekritischer Manier heißt, daß ein jedes reklamierte , Interesse des Gemeinwohls“ innerhalb einer klassengespaltenen Gesellschaft eine Fiktion ist". Schriften an zwei Fronten kämpfen. Einmal gegen den Rechtspositivismus von Hans Kelsen, der in den Augen von Fraenkel der nationalsozialistischen Pervertierung des Rechts nichts normativ substantielles entgegen zu setzen hatte; und auf der anderen Seite gegen den Machtpositivismus von Carl Schmitt, der der nationalsozialistischen Pervertierung des Rechts nichts normativ substantielles entgegen setzen wollte. Beide Positionen leugneten die materielle Bindung des Rechts an übergeordnete Werte und waren deshalb laut Fraenkel mitverantwortlich für den Zusammenbruch der Weimarer Republik. ${ }^{10}$ Während des Nationalsozialismus warf er in seinem Doppelstaat dem Regime einen ausschließlich ideologischen Gebrauch der Rede vom ,Gemeinnutzen" vor. ${ }^{11}$ Auch nachdem Fraenkel sich nach seiner Rückkehr in die Bundesrepublik (1950) mit dem Begriff des Gemeinwohls angefreundet hatte, blieben Spuren seines damaligen Mißtrauens erhalten, etwa wenn er an einer Stelle eine Bemerkung Gustav Radbruchs aus der Weimarer Zeit zitierte, in der dieser das Gemeinwohl als die große Lebenslüge unserer Zeit bezeichnet ${ }^{12}$ oder wenn er fragte: „läuft nicht jeder, der heute noch mit diesem Begriff operiert, Gefahr, sich dem Vorwurf des Ideologieverdachtes auszusetzen und ein Opfer dessen zu werden, was vor mehr als einem halben Jahrhundert als die große Lebenslüge des Obrigkeitsstaates bezeichnet worden ist?" ${ }^{13}$ Das Mißtrauen gegen den ideologischen Gebrauch dürfe nicht abgelegt werden: „Die Politikwissenschaft hörte auf, eine Wissenschaft zu sein, wenn sie das Wort ,Gemeinwohl' unkritisch verwenden wollte ". ${ }^{14}$

\footnotetext{
9 Fraenkel 1928, S. 286.

10 Vgl. zur Stellung der Argumente Fraenkels in der Debatte der Weimarer Staatsrechtslehre: Buchstein 2000.

11 Vgl. Fraenkel 1941, S. 112 f.

12 Vgl. Fraenkel 1967a, S. 285.

13 Fraenkel 1966b, S. 272.

14 Fraenkel 1963b, S. 339.
} 
Wenn sich Fraenkel in seinem Spätwerk dennoch für eine Rehabilitierung des Gemeiwohlbegriffs einsetzte, wollte er es zugleich gegen dessen totalitäre Pervertierung wie dessen vollständige ideologiekritische Auflösung retten. Eine Ansporn für seine Bemühungen mochte Fraenkel, der 1938 in die USA emigrierte, von seine Erfahrungen in der amerikanischen Wissenschaftskultur verspürt haben. Denn ungeachtet der behavioralistischen Revolution in der amerikanischen Politikwissenschaft war der Begriff des Gemeinwohls oder des öffentlichen Interesses im Denkhorizont der dortigen Faches in den 40er, 50er und bis zur Mitte der 60er Jahre des 20. Jahrhunderts fest justiert.

\section{Zwei Modi der pluralistischen Rousseau-Kritik}

Die Unsicherheit der Politikwissenschaft im Umgang mit dem Begriff des Gemeinwohls gesteht Fraenkel in seinem Spätwerk zunächst unumwunden ein. Er bezeichnet das Gemeinwohl zwar als einen „unentbehrlichen Begriff“, weist aber auch darauf hin, daß dessen Bedeutung ,weitgehend ungeklärt ${ }^{\text {"15 }}$ sei. In Anschluß an das Buch The Public Interest des amerikanischen Politikwissenschaftlers Glendon Schubert (1960) unterscheidet er drei Gemeinwohlkonzeptionen in der zeitgenössischen amerikanischen Politikwissenschaft: $^{16}$

- eine idealistische Theorie, die von der Vorstellung eines vorgegebenen Wohles Aller ausgehe,

- eine rationalistische Theorie, die das Gemeinwohl mit der öffentlichen Meinung identifiziere sowie

- eine realistische Theorie, die das Gemeinwohl mit der Resultante im Kräfteparallelogramm der Gruppenwillen idendifiziere.

Während Glendon Schubert schon einen neuen Trend in der amerikanischen Gemeinwohl-Debatte einleitete, in dessen Folge der Begriff seit Mitte der 1960er Jahre mit ideologiekritischen und soziologischen Argumenten in Auflösung geriet und später vom mainstream des Faches schlicht suspendiert wurde, nahm Fraenkel dessen Studie zum Anlass, den Gemeinwohlbegriff neu zu konzeptionieren. Zu diesem Zweck stellt er zunächst die idealistische und die realistische Gemeinwohlkonzeption (im Sinne Schuberts) gegenüber, um dann aus diesem Kontrast eigene Vorstellungen zu gewinnen. Fraenkels Auseinandersetzung mit den beiden genannten Positionen läßt sich am besten anhand seiner Kritik an Rousseau verdeutlichen. Sie geht in zwei Richtungen, eine materiale und eine prozedurale.

\subsection{Fraenkels Rousseau-Kritik in materialer Perspektive}

Fraenkel entfaltet seine materiale Kritik an Rousseau in dem Aufsatz ,Die repräsentative und die plebiszitäre Komponente im demokratischen Verfassungsstaat ${ }^{*}$ aus dem

${ }^{15}$ Fraenkel 1960 b, S. 58.

16 Vgl. ebd., S. 58. 
Jahre 1958. Danach gibt es zwei Idealtypen von Regierungssystemen, das plebiszitäre und das repräsentative System. Das plebiszitäre System gehe „von der stillschweigenden Voraussetzung eines einheitlichen Volkswillens aus, von dem a priori angenommen wird, daß er mit dem Gesamtinteresse identisch ist ${ }^{4} .{ }^{17}$ Das plebiszitäre System basiere auf zwei naturrechtlich begründeten Axiomen, dem individuellen politischen Beteiligungsrecht und der Volkssouveränität. Dem plebiszitär gebildeten Staatswillen werde keine andere Schranke auferlegt als die Verpflichtung, diese beiden Voraussetzungen politischer Herrschaftsausübung zu respektieren. Fraenkel zufolge könne sich auf diese Weise bei einer freien Entscheidung des Volkes im Ergebnis ,jedwedes Interesse, d. h. auch ein vom objektiven Blickpunkt aus betrachtet, beliebiges Interesse zum Gesamtinteresse erklären“. ${ }^{18}$ Das Bekenntnis zum plebiszitären Legitimationsmodus ,schließt die Anerkennung eines originär präexistenten Gemeinschaftswohls aus" ${ }^{\text {“ }}{ }^{19}$ Mit anderen Worten: der plebiszitäre Legitimationsmodus bietet für Fraenkel keine Schranken gegen einseitige und grob ungerechte politische Entscheidungen.

Anders und deshalb besser sei der repräsentative Legitimationsmodus. Er geht von der These eines ,vorgegebenen und objektiv feststellbaren Gesamtinteresses und der Hypothese aus, daß der Wille des Volkes auf die Förderung des Gesamtinteresses gerichtet sei (Hypothetischer Volkswille)“. ${ }^{20}$ Für Fraenkel geht das Repräsentativsystem von dem Axiom eines ,naturrechtlich basierten, (weder aus einem Kollektivwillen noch gar aus einem individuellen ableitbaren und deshalb) originären Gesamtinteresse aus" ${ }^{61}$ Im repräsentativen System werde die Entscheidung über den Staatswillen primär unter der Berücksichtigung der Erwägung getroffen, „dem Gesamtinteresse eine tunlichst große und ungehinderte Chance der Entfaltung zu geben“. ${ }^{22}$ Die reine Form des Repräsentativsystems sieht Fraenkel in der französischen Verfassung von 1791 realisiert, nach der die nur ihrem Gewissen verantwortlichen Abgeordneten der Nationalversammlung - und zwar nur sie - „die volonté générale, zu deutsch den hypothetischen Willen des Volkes - das heißt aber das Gemeinwohl ‘23 repräsentieren.

Pointiert läßt sich die Unterscheidung, die Fraenkel vornimmt, folgendermaßen formulieren: während der plebiszitäre Legitimationsmodus zwei prozedurale Axiome (individuelle politische Partizipationsrechte und Volkssouveränität) als unverrückbares Naturrecht anerkennt, orientiert sich der repräsentative Legitimationsmodus an einem naturrechtlich vorgegebenen materiellen Gehalt politischer Entscheidungen. Im Konfliktfall obliegt im plebiszitären Modell das prozedurale Moment über das inhaltliche, im repräsentativen Modell ist es umgekehrt. Nach der Logik dieser Unterscheidung kann für Fraenkel allein der repräsentative Legitimationsmodus verhindern, daß es zu politischen Entscheidungen kommt, die einseitig die Interessen nur eines Teils der Gesellschaft berücksichtigen.

\footnotetext{
17 Fraenkel 1958a, S. 154.

18 Ebd., S. 156.

19 Ebd.

20 Ebd., S. 153.

21 Ebd., S. 155

22 Ebd.

23 Ebd, S. 154.
} 
Ich habe Fraenkels Überlegungen zum plebiszitären und repräsentativen Legitimationsmosdus ausführlich herangezogen, weil sich darin zweierlei zeigt. Zum einen verdeutlichen sie, warum Fraenkel, trotz seiner bekannten Forderung nach einer Mischung plebiszitärer und repräsentativer Momente in der Demokratie ${ }^{24}$ sich letztlich als klarer Verfechter des Vorrangs des Repräsentativsystems zu erkennen geben muß. Zweitens - und in unserem Zusammenhang wichtiger - belegen die referierten Überlegungen, daß Fraenkel den Anspruch erhebt, daß die pluralistische Demokratietheorie über materiale Kriterien bei der Bewertung von politischen Entscheidungen, die den Gemeinwohlstatus reklamieren, verfügt. "Wenn wir von Gemeinwohl reden" so Fraenkel, „denken wir nicht in Kategorien der Macht, sondern in Kategorien des Rechts und der Gerechtigkeit: wir bewegen uns nicht im Bereich des Seienden, sondern des SeinSollenden. Gemeinwohl ist keine soziale Realität, sondern eine regulative Idee“ ${ }^{25}$

Für Fraenkel ergeben sich aus seinen bisherigen Überlegungen politische und methodologische Konsequenzen, die den Habitus politischer Akteure in der modernen Demokratie und die den Habitus von Politikwissenschaftlern betreffen. Von den politischen Akteuren in der pluralistischen Demokratie - er richtet sich vor allem an Parteien und Verbände - verlangt er, daß sie den Bezug auf das Gemeinwohl als ,immanente Schranken ${ }^{، 26}$ ihrer Aktivitäten immer im Blick behalten sollen und ihre Interessen nicht überreizen. Politologen sind zu einer vergleichbaren Zurückhaltung aufgerufen. Sie sollen die Grenzen der soziologischen Verflüssigung politischer Ideen anerkennen. Wohl auch selbstkritisch auf seine eigene ideologiekritische Position während der Weimarer Republik bezogen schreibt er: „[W]enn alle Ideen nichts anderes als Ideologien darstellen, kann es notwendigerweise auch nicht das Minimum eines an der Idee eines Gemeinwohls ausgerichteten Gemeinschaftsdenkens geben, das unerläßlich ist, um einen pluralistischen Staat lebensfähig zu erhalten. ${ }^{227}$ Die zitierte Bemerkung kommt einer Selbstnötigung Fraenkels gleich, denn er übernimmt damit die Begründungslast für die normative Geltung der das Gemeinwohl fundierenden Ideen; doch bevor ich der Fundierungs-Frage weiter nachgehe, soll zunächst der zweite Aspekt der Fraenkelschen Gemeinwohlkonzeption herausgestellt werden.

\subsection{Fraenkels Rousseau-Kritik in prozeduraler Perspektive}

Auch in diesem Punkt rekurriert Fraenkel auf Rousseau als Abgrenzungsfolie, besonders prägnant in seinem Beitrag Der Pluralismus als Strukturelement der freiheitlichrechtsstaatlichen Demokratie aus dem Jahre 1964. Fraenkel zufolge lassen sich die beiden deutschen politischen Systeme der damaligen Zeit, die demokratische und pluralistische Bundesrepublik und die totalitäre DDR, anhand der ihnen jeweils unterliegenden Gemeinwohlkonzeptionen unterscheiden. Fraenkel zufolge gehen totalitäre Diktaturen „von der Hypothese eines eindeutig bestimmbaren vorgegebenen Gemeinwohls

\footnotetext{
${ }^{24}$ Vgl. Fraenkel 1958a, S. 159, 202.

25 Fraenkel 1960b, S. 61.

26 Ebd., S. 64.

27 Fraenkel 1963b, S. 346.
} 
aus". ${ }^{28}$ Von diesem Gemeinwohl wird unterstellt, „es sei ausreichend detailliert, um [...] als politisches Aktionsprogramm verwertet zu werden". ${ }^{29}$ Das Gemeinwohl liegt danach ,,a priori ${ }^{\star 60}$ längst vor aller gesellschaftlichen Auseinandersetzung unzweifelhaft fest. In der Logik eines solchen Ansatzes liegt es, daß seine Anhänger die Vorstellung hegen, daß Divergenzen bei der Bestimmung des Gemeinwohls ein Erkenntnisproblem sei. In der Logik eines solchen Ansatzes liegt es Fraenkel zufolge meist auch, daß seine Anhänger sich selbst vor möglichen Erkenntnisschranken bewahrt sehen. Als besonders begnadete Politiker oder Staatsmänner reklamieren sie, nicht nur das objektive Gemeinwohl zu erkennen, sondern auch, daß sie es sind, die es notfalls gegen Widerstand in der Gesellschaft realisieren müssen. Ein Gemeinwohl, daß der Gesellschaft von selbst-auserwählten Personen aufgezwungen werden soll, nennt Fraenkel ,heteronom legitimiert".31

Als mögliche Erkenntnisquellen für ein solches Gemeinwohl a priori haben Fraenkel zufolge in der Geschichte der politischen Ideen ganz unterschiedliche Ideologien fungiert. Es kann der Anspruch auf wissenschaftlichen Rationalismus, religiöse Inspiration, Rassenwahn oder auch die Einsicht in den objektiven Geschichtsverlauf sein, aus denen Personen das Recht ableiten, das Gemeinwohl a priori der Konsultation anderer Gesellschaftsangehöriger erkannt zu haben. ${ }^{32}$ Alle diese Gedankengänge führen nach Fraenkel zum Totalitarismus.

Erneut ist es Rousseau, den Fraenkel als Ur-Vater einer solchen verfehlten Gemeinwohlkonzeption benennt. In verschiedenen Aufsätzen hat er sich der Mühe unterzogen, den Nachweis zu erbringen, wie sehr der moderne Totalitarismus von Rousseaus Gemeinwohlkonzept durchdrungen sei. Fraenkel wiederholt hier Interpretationen, die er bereits in der Weimarer Republik gegen eine ,absolutistische Demokratietheorie“ Rousseaus vorbrachte. ${ }^{33}$ In seinem Spätwerk kommt noch die zustimmende Aufnahme der totalitarismustheoretischen Rousseau-Deutung Jacob Talmons hinzu. Fraenkel zufolge sei Rousseau der Ansicht gewesen, das Gemeinwohl sei objektiv erkennbar und er sei nicht mißverstanden worden, als in seinem Namen die Artikulation partikularer Interessen verboten wurde. Rousseaus Gemeinwohlkonzeption verlange ein Höchstmaß gesellschaftlicher Homogenität, die notfalls auch mit terroristischen Mitteln vom législateur durchgesetzt werden muß. ${ }^{34}$

Die pluralistische Gemeinwohlkonzeption beschreibt Fraenkel in Kontrast dazu als offener: „Der Pluralismus beruht [...] auf der Hypothese, in einer differenzierten Gesellschaft könne im Bereich der Politik das Gemeinwohl lediglich a posteriori als das Ergebnis eines delikaten Prozesses der divergierenden Ideen und Interessen der Grup-

28 Fraenkel 1964 b, S. 300.

29 Ebd.

30 Ebd., S. 297.

31 Fraenkel 1963 b, S. 364.

32 Vgl. Fraenkel 1960b, S. 58-61.

33 Vgl. Fraenkel 1929, S. 359 f.; 1932, S. 499 f., 507 f.

34 Vgl. Fraenkel 1958b, S. 212 f.; 1964b, S. 307-324; 1966b, S. 265 ff.; 1967, S. 279 ff.; 1969 , S. 338 . 
pen und Parteien erreicht werden ${ }^{\text {“ }}{ }^{35}$ Das Gemeinwohl wird der Gesellschaft nicht von einer Minderheit aufgezwungen, sondern entsteht im Prozeß der offenen Konfliktaustragung in der Gesellschaft. Zu diesem Prozeß gehören Verhandlungen, Diskussionen und Kompromisse. ${ }^{36}$ Die politischen Institutionen der westlichen Demokratie - Parlament, Parteien, Verbände, Öffentlichkeit - sind die Orte, in denen in öffentlicher Kommunikation das Gemeinwohl erst entsteht. Viel kommt deshalb darauf an, daß die demokratischen Institutionen für einen solchen Proze $\beta$ offen genug organisiert sind. Fraenkel bezeichnet Gemeinwohl, das am Ende eines öffentlichen Kommunikationsprozesses steht, als ,autonom legitimiert“ ${ }^{37}$ da es aus der Mitte der Gesellschaft hervorgebracht und ihr nicht von einer mit einem Erkenntnisprivileg ausgestatteten Minderheit aufgezwungen wird.

Die beiden konkurrierenden Gemeinwohlkonzeptionen taugen für unterschiedliche Gesellschaftsmodelle. Während das heteronome Gemeinwohl auf eine homogene $\mathrm{Ge}-$ sellschaft drängt, kann sich das autonome Gemeinwohl erst in einer heterogenen $\mathrm{Ge}-$ sellschaft entfalten. Pluralität und Kommunikation ist für Fraenkel Bedingung der Herstellung eines echten Gemeinwohls. Er projiziert diese Idee zurück bis nach England im 18. Jahrhundert: „Nur weil das Parlament dem heterogenen Charakter der Gesellschaft Ausdruck verlieh, galt es als geeignet, dem Gemeinwohl zu dienen" ${ }^{38}$

Auch wenn das Gemeinwohl a posteriori ein Ergebnis gesellschaftlicher Auseinandersetzungen protokolliert, ist es inhaltlich nicht beliebig. Hier wiederholt Fraenkel Punkte, die er bereits gegen seine erste (und prozedurale) Rousseau-Lesart vorgetragen hatte. Das Ergebnis politischer Prozesse verdient nur dann das Gütesiegel ,Gemeinwohl', wenn es bestimmte inhaltliche Mindestqualifikationen erfüllt.

\subsection{Materiale und prozedurale Aspekte in Fraenkels Gemeinwohlkonzeption}

Unabhängig davon, wie man Fraenkels Rousseau-Interpretationen im einzelnen beurteilt - es ist deutlich geworden, daß Fraenkels Gemeinwohlkonzept sowohl eine prozedurale und wie eine materiale Seite hat. Das prozedurale Moment ist gegen Positionen gerichtet, die über so genaue Vorstellungen des Gemeinwohls verfügen, daß sie den demokratischen Kommunikations- und Willensbildungsprozeß nur noch als unnötige Störung wahrnehmen können. Das materiale Moment ist gegen Positionen gerichtet, die lediglich korrekte Prozeduren kennen und deshalb jedes Ergebnis des politischen Prozesses als Gemeinwohl anzuerkennen bereit sind. ,Gemeinwohl' steht für Fraenkel also als Qualitätssiegel für eine politische Entscheidung, wenn sie gleichermaßen zwei Bedingungen erfült: Sie ist (1) in einem makellosen Verfahren politischer Willensbildung zustande gekommen, und sie erfüllt darüber hinaus (2) ein angebbares Maß materieller Mindeststandards. Eine der wenigen pointierten Definitionen Fraenkels von Gemein-

\footnotetext{
${ }^{35}$ Fraenkel 1964b, S. 300.

${ }^{36}$ Fraenkel 1969, S. 330.

${ }^{37}$ Fraenkel 1964b, S. 325.

${ }^{38}$ Fraenkel 1960b, S. 56.
} 
wohl lautet: „Gemeinwohl stell(t) die Resultante dar, die sich jeweils aus dem Parallelogramm der ökonomischen, sozialen, politischen und ideologischen Kräfte einer Nation dann ergibt, wenn ein Ausgleich angestrebt und erreicht wird, der [erste Bedingung H. B.] objektiv den Mindestanforderungen einer gerechten Sozialordnung entspricht und [zweite Bedingung - H. B.] subjektiv von keiner maßgeblichen Gruppe als Vergewaltigung empfunden wird“. ${ }^{39}$

Die angeführte Textpassage erfreut sich in der einschlägigen Sekundärliteratur häufiger Zitation. Von vielen Kritikern aus den 1960er und 1970er Jahren wurde einseitig nur die Parallelogramm-Metapher aufgegriffen und Fraenkels Gemeinwohlbegriff der Vorwurf gemacht, daß seine Pluralismustheorie der Dynamik sozialer, ökonomischer, sozialer und ideologischer Kräfte blind vertraue und dabei die Tatsache, daß diese Einflußfaktoren zum einen auf gesellschaftlich ungleich verteilte Machtpositionen basieren und zum anderen unterschiedlich kompatibel sind mit den Strukturen des politischen Systems, übersähe. ${ }^{40}$ Die beiden in dem Zitat formulierten Bedingungen belegen indes, daß die damaligen Kritiker Fraenkels Neopluralismustheorie offensichtlich mit Tendenzen in der zeitgenössischen amerikanischen Pluralismustheorie, die sich durch ein selbstgewisses Prozeßvertrauen in die bestehenden demokratischen Verfahren auszeichneten, ${ }^{41}$ verwechselt haben.

Fraenkels Versuch, materiale und prozedurale Aspekte miteinander zu verbinden, erwecken bei oberflächlicher Lektüre den Eindruck der Widersprüchlichkeit. Angesichts seiner klaren inhaltlichen Bestimmungen des Gemeinwohls in seiner Kritik an einem bloß prozeduralen Gemeinwohlverständnis scheint sich Fraenkels Konzeption in einen argumentativen Zirkel zu begeben. Um nicht jedem beliebigen Ausgang politischer Willensbildungsprozesse automatisch das Prädikat "gemeinwohlorientiert“ umhängen zu müssen, hatte Fraenkel subjektive, kulturbedingte und schließlich auf einer letzten Ebene objektive Wertmaßstäbe als Bewertungskriterien eingeführt. Diese Wertmaßstäbe nun sollen - wie er es zuvor Rousseaus volonté générale noch vorgeworfen hatte - dem politischen Streit enthoben und von unabdingbarer Gültigkeit sein. Die von Fraenkel aufgemachte Differenz zwischen einem Gemeinwohl a priori und einem Gemeinwohl a posteriori gerät an dieser Stelle ins Schwimmen.

Dieses Problem läßt sich meines Erachtens im Rahmen von Fraenkels Denken ,immanent" lösen, wenn man die beiden Gemeinwohlkonzeptionen auf Ebenen unterschiedlicher Abstraktionshöhe ansiedelt. Bei der klassischen Theorie der Demokratie handelt es sich um die "Existenz eines vorgegebenen Volkswillens“ ${ }^{42}$ der den Status eines „Aktionsprogramm[es] praktischer Politik ${ }^{\star 43}$ hat. Das Gemeinwohl a priori ist so

\footnotetext{
39 Fraenkel 1960a, S. 34; oder auch: Nur dann könne etwas als Gemeinwohl anerkannt werden, wenn „die Auseinandersetzungen unter Einhaltung der Regeln eines fair-play geführt werden und die Ergebnisse der Auseinandersetzungen sich im Rahmen der Mindesterfordernisse der sozialen Gerechtigkeit bewegen" (Fraenkel 1960b, S. 65).

40 Vgl. Narr 1969, Eisfeld 1972. So fälschlicherweise auch wieder Offe 2002, S. 73.

${ }^{41}$ Zur damaligen Kritik an diesen Vorstellungen vgl. Mills 1956, Scharpf 1970, S. 87 f., Offe 1972, S. $65 \mathrm{ff}$.

${ }^{42}$ Fraenkel 1964a, S. 87.

${ }^{43}$ Fraenkel 1967a, S. 285.
} 
konkret und detailliert, daß es direkt implementiert werden kann. Anders beim Gemeinwohl a posteriori. Dies bleibt Fraenkel zufolge in weiten Teilen der konkreten Ausgestaltung durch politische Akteure überlassen und ist lediglich in den Rahmen „einer vorgegebenen Wertordnung “44 eingehängt. Dieser Rahmen ist so unkonkret, daß er der situativen Konkretion durch Politik bedarf. Abgesteckt sind lediglich die Grenzen. Diese Grenzen aber sind absolut, und insofern ist die Unterscheidung der Legitimationsmodi der beiden Gemeinwohlkonzeptionen als ,autonom' vs. ,heteronom ' nicht ganz zutreffend. Auch das autonom legitimierte Gemeinwohl a posteriori verfügt über ein nicht reduzierbares Moment des Heteronomen.

In seinen Schriften hat sich Fraenkel mehr mit dem prozeduralen Aspekt befaßt. Dies hat zeitbedingte Gründe. Ein Grund ist politischer Natur: Fraenkel hält diesen Aspekt in Deutschland für sträflich unterentwickelt und möchte mit seinen Schriften zu einer Auflösung homogenisierender Gemeinwohlvorstellungen beitragen. Vor dem Hintergrund des Nachwirkens der nationalsozialistischen Doktrin ,Gemeinnutz geht vor Eigennutz' will Fraenkel das Konzept des Gemeinwohls nicht aufgeben, sondern epistemisch relativieren, genetisch dynamisieren und inhaltlich mit Mindeststandards versehen. Der zweite Grund ist wissenschaftlicher Natur: Fraenkel ist es in seinen Arbeiten nicht gelungen, den normativen Kern des materialen Gemeinwohlaspekts überzeugend darzulegen - auf diesen Punkt werde ich weiter unten im fünften Abschnitt näher eingehen.

\section{Das Gemeinwohl im politischen System der pluralistischen Demokratie}

Das Gemeinwohl und die Gemeinwohlorientierung politischer Akteure - der Gemeinsinn - sind ein zentrales Moment in der normativen Skizze, die Fraenkel vom politischen System einer gut funktionierenden pluralistischen Demokratie entwirft. Dieses normative Modell soll die notwendigen Voraussetzungen benennen, um die von Carl Schmitt so eindringlich dargelegte dynamischen Kraft von Konflikten und ihren potentiell zentrifugalen Effekten in pluralistischen Demokratien unter Kontrolle zu halten.

Dem Gemeinwohl kommt in diesem Bild Fraenkels eine zentrale Stellung zu, wie aus einer Definition von 1963 hervorgeht: „Unter dem ,Gemeinwohl“ wird [...] eine in ihrem Kern auf einem als allgemein gültig postulierten Wertkodex basierende, in ihren Einzelheiten den sich ständig wandelnden ökonomisch-sozialen Zweckmäßigkeitserwägungen Rechnung tragende regulative Idee verstanden, die berufen und geeignet ist, bei der Gestaltung politisch nicht kontroverser Angelegenheiten als Modell und bei der ausgleichenden Regelung politisch kontroverser Angelegenheiten als bindende Richtschnur zu dienen" ${ }^{45}$ Fraenkels erster großer Strich in dem normativen Gemälde ist eine Unterscheidungslinie zwischen zwei Sektoren: einem kontroversen Bereich, in dem die

44 Fraenkel 1964a, S. 87.

45 Fraenkel 1963b, S. 339. 
autonomen Verbände um Interessen und Einfluß ringen, und einem Bereich von Internalisierungen, die den nicht-kontroversen Sektor der Gesellschaft bilden. Fraenkel schließt mit dieser Aufteilung (oder besser: Eingrenzung) an einen Gedanken an, den er schon während der Weimarer Republik publizierte. In dem Aufsatz ,Um die Verfassung ${ }^{6}$ konzipierte er einen historisch gewachsenen unstreitigen Sektor und einen streitigen Sektor, den er angesichts des zerrütteten Zustandes der Republik sorgfältig zu begrenzen sucht. $^{46}$

In seinem Spätwerk nimmt Fraenkel diesen Gedanken erneut unter Rekurs auf Robert A. Dahls A Preface to Democratic Theory (1956) auf. In einer funktionierenden westlichen Demokratie spielt sich Fraenkel zufolge „der weitaus größere Teil obrigkeitlicher und gesellschaftlicher Betätigung ${ }^{647}$ im Raum des nicht-kontroversen Sektors ab. Damit Demokratien langfristig und ungestört erhalten bleiben, muß dieser Sektor den überwiegenden Anteil des gesellschaftlichen Lebens ausfüllen. Nur unter dieser Bedingung ist die Gesellschaft dem Streit der Meinungen im kontroversen Bereich gewachsen. Die Grenzlinie zwischen den beiden Sektoren liegt nicht ein für alle Male fest, sie ist „ständigen Verschiebungen unterworfen“" ${ }^{48}$ Gerät die Routine des staatlichen und gesellschaftlich-politischen Verhaltens in Widerspruch zu den Grundprinzipien der Gesellschaftsordnung, können diese Dysfunktionalitäten in den kontroversen Sektor gerückt und korrigiert werden. Umgekehrt können erreichte Lösungen ehemals kontroverser Fragen in den nicht-kontroversen Bereich einfließen und in ihm Bestand erhalten.

Fraenkel unterscheidet in diesem Zusammenhang zwischen einem genuinen, einem derivativen und einem originären Gemeinwillen. Ein genuiner Gemeinwille liege vor, wenn die in einem vorgegebenen Wertkodex enthaltenen Normen und Regeln des menschlichen Umgangs so generell anerkannt sind, daß sie „als selbstverständlich hingenommen und befolgt werden“. ${ }^{49}$ Die Bestandteile des genuinen Gemeinwillens werden als so unproblematisch anerkannt, daß sie weder in der Vergangenheit kontrovers waren, noch in der Zukunft kontrovers sein werden. Die beiden Unterfälle sind der originäre und der derivative Gemeinwille. Um den erstgenannten handelt es sich, wenn die Kontroversen, die einst dem originären Gemeinwillen zugrundegelegen haben, ,total vergessen sind" ${ }^{50}$ so das dies faktisch dem genuinen Gemeinwillen nahekommt. Um einen derivativen Gemeinwillen handelt es sich, wenn im Bewußtsein der politischen Akteure die vergangenen politischen Kämpfe und Kompromisse um seine Findung und sei es auch ,vage“ - erinnert werden. Die beiden weiteren Abstufungen bezeichnet Fraenkel als „konsolidierte öffentliche Meinung“ und „fluide öffentliche Meinung“. Hat sich in der ersten eine Art öffentliche Akzeptanz zu einem bestimmten Thema herausgeschält, so liegt letztere bereits im kontroversen Sektor. Von besonderer Bedeutung für Fraenkels Konzeption des Gemeinwohls sind der genuine und der originäre Gemeinwille. Beide müssen inhaltlich bestimmten Werten entsprechen.

\footnotetext{
${ }^{46}$ Vgl. Fraenkel 1932a, S. 505.

47 Fraenkel 1963a, S. 248.

48 Ebd., S. 249

49 Ebd., S. 250.

${ }^{50}$ Ebd.
} 
Die Sicherungsfunktion des nicht-kontroversen Sektors ist nicht nur funktional bedingt, sondern wird durch eben diese spezifischen Inhalte erst ermöglicht. Die Inhalte werden von Fraenkel allerdings recht diffus umschrieben und zudem erst relativ spät ab 1960 - ausformuliert. Nur mit einem gewissen Mut zur interpretatorischen Zuspitzung läßt sich aus Fraenkels verstreuten Überlegungen zum nicht-kontroversen Sektor eine Art Systematik rekonstruieren. Der Großteil der Bestandteile des ,consensus omnium“ rangiert danach , unterhalb der kollektiven Bewußtseinsschwelle ${ }^{c 5}{ }^{51}$ Er ist ein empirisches Phänomen und wohl am ehesten zu übersetzen mit dem Topos ,Politische Kultur' ${ }^{52}$ Er besteht aus sedimentierten geschichtlichen Erfahrungen. Diese Erfahrungen sind $\mathrm{zu}$ Wertvorstellungen geronnen, die das politische Denken der Bevölkerung bestimmen. Hierzu zählt Fraenkel: die Spielregeln des politischen Wettbewerbs, die Verpflichtung auf die Verfahrensregeln der Willensbildung und diverse Grundprinzipien ,gesitteten menschlichen Zusammenlebens ${ }^{53}$. Später differenziert Fraenkel die inhaltlichen Bestandteile weiter aus und zählt zu ihnen Sprache, Kulturbewußtsein, Tradition, Abstammung, Verfassungsvorstellungen, aber auch Übereinstimmungen im wirtschaftlichen Bereich. Zum ,consensus omnium' gehören des weiteren eingeschliffene Verhaltensweisen im politischen Alltag. ${ }^{54}$

Fraenkel geht davon aus, daß die Existenz eines nicht-kontroversen Bereichs zwar eine notwendige, aber noch keine hinreichende Bedingung einer gut funktionierenden westlichen Demokratie bildet. Fraenkel nimmt deshalb implizit eine Präzisierung des ,consensus omnium 'vor, wenn er einen spezifischen ,reflektierten consensus ${ }^{655}$ als das bewußte Element menschlichen Handelns in diesem Sektor ortet. Die Funktion des reflektierten consensus besteht neben der Integration der divergierenden Willen vor allem in der Legitimation getroffener Vereinbarungen. Er gilt Fraenkel als ,unerläßlich, um als tragfähige Basis für den Abschluß der allfälligen Kompromisse zu dienen“. ${ }^{\circ 6}$ Der Inhalt des reflektierten consensus besteht aus einem allgemeinverbindlichen Wertkodex. Zum Wertkodex dürfen drei Elemente gerechnet werden:

(a) Verfahrens- und Verhaltensregeln. Zu ihnen gehören nicht nur die Normen der Verfassung und ihrer Ausführungsgesetze, sondern auch die gesellschaftlich sanktionierten Gesetze des Fair-Play und des Takts.

(b) Zweitens ein Set konkreter politischer und sozialer Prinzipien. Fraenkel zählt als Teile auf: die Anerkennung der Volkssouveränität, soziale Gerechtigkeit, das Prinzip Gleichheit vor dem Gesetz, traditionelle Freiheitsrechte und Überparteilichkeit der Justiz. Diese Prinzipien sieht Fraenkel als in der historischen Entwicklung der westlichen Demokratien herausgebildet und unhintergehbar an und siedelt sie im originären oder genuinen Gemeinwillen an.

\footnotetext{
51 Ebd., S. 254.

52 Vgl. Massing 1979, S. 121, Buchstein 1998.

53 Fraenkel 1966b, S. 275.

54 Vgl. Fraenkel 1969, S. 354 f.

55 Fraenkel 1960b, S. 60.

56 Ebd., S. 61.
} 
(c) Deren inhaltlichen Kern bilden die „fundamentalen regulativen Ideen“. Die regulativen Ideen sind kein Bündel von Vorstellungen, das als direktes gesellschaftliches Aktionsprogramm zu verwenden wäre, denn dann wäre das Gemeinwohl ja so weit vorgegeben, da $B$ es keiner pluralistischen Entfaltung mehr bedürfte. Die regulativen Ideen sind abstrakte Prinzipien, von denen er mit „Gerechtigkeit und Billigkeit ${ }^{67}$ zwei benennt.

Der Wertkodex ist ein sowohl empirisches wie normatives Phänomen. Sein empirischer Bestandteil ist das de facto anerkannte „Minimum an Übereinstimmung“ (1960b: 61), das zum Funktionieren einer Demokratie unerläßlich ist. Dieses empirische Substrat ist flexibel und seine Konkretisierung erfolgt immer wieder neu durch die reflektierte Anpassung an sich wirtschaftlich, sozial und politisch wandelnde Gegebenheiten. Der normative Teil beruht auf seiner Anerkennungswürdigkeit, auf seiner „verpflichtenden Kraft" ${ }^{58}$ Die Anerkennungswürdigkeit geht aus vom dritten Element des Wertkodexes, in den regulativen Ideen. Ihre universelle Überzeugungskraft speist sich aus ihrer naturrechtlichen Vorgegebenheit.

Zusammengefaßt ergibt sich aus dieser Darstellung ein Gesamtbild, das einer aufgeschnittenen Zwiebel ähnelt.

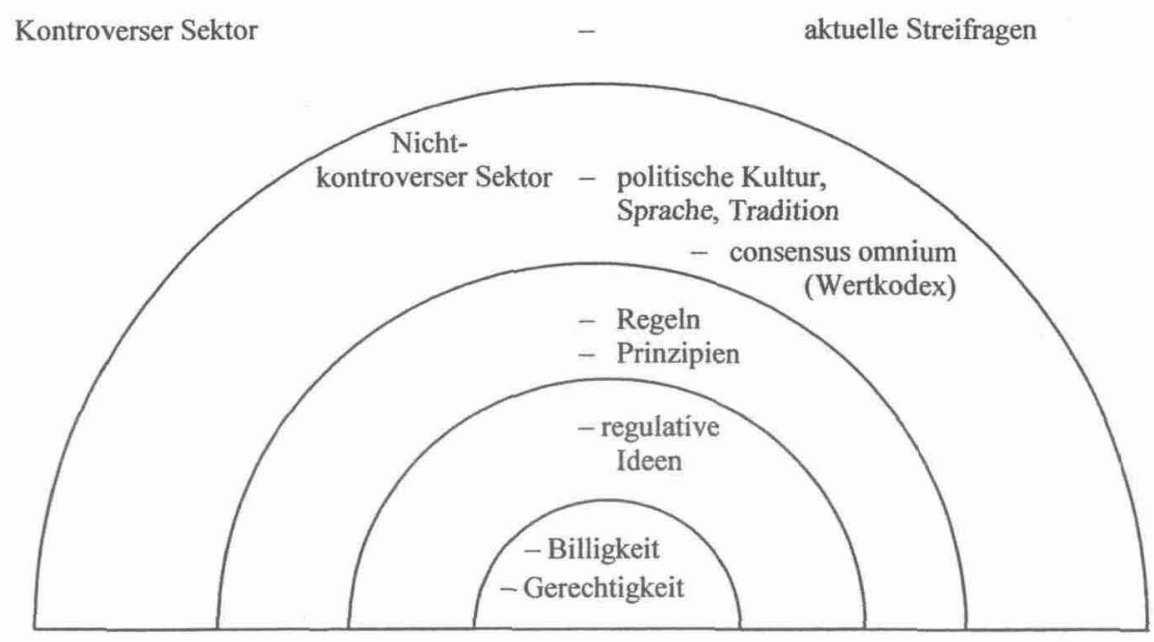

Übersicht I: Die Elemente des kontroversen und des nicht-kontroversen Sektors bei Ernst Fraenkel

\footnotetext{
${ }^{57}$ Fraenkel 1964b, S. 324.

58 Fraenkel 1960b, S. 61.
} 
Auf Unterschiede stoßen wir, wenn wir die einzelnen Schichten des nicht-kontroversen Sektors betrachten:

\begin{tabular}{|l|l|l|l|}
\hline & $\begin{array}{l}\text { Nicht-kontroverser } \\
\text { Sektor }\end{array}$ & Consensus omnium & Regulative Ideen \\
\hline Inhaltlich & diffus & präzise & abstrakt \\
\hline Bewußtseinsmäßig & unbewußt & reflektiert & reflektiert \\
\hline Epistemisch & empirisch & empirisch-normativ & normativ \\
\hline
\end{tabular}

\section{Übersicht II: Der Status der Elemente des nicht-kontroversen Sektors}

Für die Herstellung einer am Gemeinwohl orientierten politischen Entscheidung kommt den regulativen Ideen eine zentrale Bedeutung zu. Die normative Begründungslast können sie tragen, da sie auf Werten des rationalen Naturrechts basieren: „Naturrecht nennen wir ein Normensystem, das Individualrechte anerkennt, die, weil sie von der Gesetzgebung des Einzelstaates unabhängig sind, nicht zu dessen Disposition stehen“. 59

Fassen wir zusammen: Das Gemeinwohl entsteht in dem Prozeß des Ringens verschiedener gesellschaftlicher Gruppen um die Lösung kontroverser Fragen. Dieser Prozeß ist nicht voraussetzungslos, sondern bedarf der Akzeptanz nichtkontroverser Essentials durch alle beteiligten politischen Akteure. Ausgehend von der absoluten Geltung abstrakter naturrechtlicher Werte postuliert Fraenkel einen Kanon an historisch unhintergehbaren Verfahrensregeln und sozialen Prinzipien sowie eine den Konjunkturen des Zeitgeistes unterworfene und allen Beteiligten gemeinsame politische Kultur. Im nächsten Abschnitt möchte ich mich den Inhalten und der Begründung des absolut geltenden Gemeinwohlkerns widmen.

\section{Naturrecht und Gemeinwohl}

Die Absicht der beiden letzten Abschnitte war es, deutlich zu machen, wie hoch der Vertrauensscheck ist, den Fraenkel auf die Geltung naturrechtlicher Normen ausstellt. Unversehens sind seine Überlegungen zum Gemeinwohl nämlich auf der Ebene der politischen Philosophie angelangt. Angesichts der Bedeutung des Naturrechts verwundert es, daß Fraenkel zu diesem Thema nie über Postulate und Andeutungen hinausgekommen ist. Doch selbst wenn man in Rechnung stellt, daß er in Berlin eine Professur für Vergleichende Regierungslehre inne hatte und die Beschäftigung mit der naturrechtlichen Basis seiner Gemeinwohlkonzeption möglicherweise in einer Art arbeitsteiligen

\footnotetext{
${ }^{59}$ Fraenkel 1958c, S. 154.
} 
Verfahren an Kollegen der Politischen Philosophie delegiert $\mathrm{sah}^{60}$ bleiben die wenigen Überlegungen zu diesem Fundament seiner Gemeinwohlkonzeption unbefriedigend. ${ }^{\text {K }}$

Welches sind die Werte, denen Fraenkel naturrechtlichen Status zuweist? Auch wenn sie abstrakt sind, müssen sie doch inhaltlich konkret genug sein, um den Politikwissenschaftler zu befähigen, Ergebnisse politischer Prozesse auf ihre Gemeinwohlkompatibilität zu befragen. In seinen Arbeiten nennt Fraenkel immer wieder „Gerechtigkeit und Billigkeit" ${ }^{62}$ Die ,Billigkeit" wird nicht näher ausgeführt; wenig besser steht es um die die ,Gerechtigkeit'. Wir erfahren lediglich, daß Fraenkel neben Menschenwürde, Freiheit und Gleichheit eine distributive Auslegung der Gerechtigkeit favorisiert, wenn er den Grundelementen der deutschen Sozialstaatlichkeit attestiert, daß sie die „Gültigkeit von Sätzen des Naturrechts “63 ${ }^{\text {6 }}$ beanspruchen können.

Ähnlich vage bleibt Fraenkel bei der Begründung naturrechtlicher Werte. Die Option für das Naturrecht läßt sich zurückverfolgen bis in seine Arbeiten der 1920er Jahre. In seinem weiteren politikwissenschaftlichen Werk weitet er den personellen Trägerkreis naturrechtlicher Gedankengänge sukzessive aus: Sprach er in den 1920er Jahren zuerst von einem klassenspezifischen Naturrecht, so wurde daraus später die antifaschistische Front der Naturrechtler. In den Arbeiten nach 1945 ist das Naturrecht bei Fraenkel zu einem allgemeinen Prinzip des Okzidents aufgestiegen. ${ }^{64}$ In seiner ersten Berliner Gastvorlesung über den Korea-Konflikt heißt es: Im Widerstand gegen einen zynisch mißverstandenen Gesetzespositivismus sowie gegen ein (gemeinschaftliches) sentimental mißverstandenes Naturrecht solle die moderne Rechtsphilosophie ein tragfähiges modernes Naturrecht begründen. ${ }^{65}$ Fraenkel versuchte sich einige Jahre später in einem Vortrag selbst an einer solchen rechtsphilosophischen Skizze. ${ }^{66}$ Das moderne Naturrecht sei ein Konglomerat aus vier Quellen: dem religiösen Glauben im katholischen

${ }^{60}$ So auch Detjen: „Man kann Fraenkel nicht als philosophischen Kopf bezeichnen, dem es um die Reflexion letzter Voraussetzungen normativer Ideenkomplexe gegangen ist" (Detjen 1988, S, 626).

61 Oder, wenn man es freundlicher ausdrücken möchte: sie sind „nicht leicht zu verstehen“ (Massing 1979, S. 139).

62 Fraenkel 1964b, S. 324.

${ }^{63}$ Fraenkel 1960b, S. 50.

${ }^{64}$ Schon in seiner Weimarer Schrift Zur Soziologie der Klassenjustiz vertrat Fraenkel naturrechtliche Positionen: Der Befreiungswille des Proletariats beruhe nicht auf der Einsicht in historische Gesetzmäßigkeiten, sondern sei motiviert durch das "naturrechtliche Rechtsbewußtsein“ (1927: S. 202, auch S. 180-182, 206) der Arbeiterklasse. Das Naturrecht ist dem jungen Fraenkel zufolge kein einmaliges historisches ,Ereignis', sondern vielmehr eine soziologisch begründete Dauererscheinung: Es ist der Rechtfertigungsgrund der unterdrückten Klassen. In seinen letzten Schriften vor dem Zusammenbruch der Republik appelliert er geradezu verzweifelt, daß das Bürgertum sich doch auf „ewigen Werte“ (1932c: S. 591, auch 1932b: S. 584) demokratischer, sozialer und humanitärer Ideale besinnen möge. Während des Nationalsozialismus und der in der Emigration verfestigt sich Fraenkels Option für das Naturrecht weiter. Dem faschistischen gemeinschaftlichen Naturrecht hält er in seinem Doppelstaat in drei Buchkapiteln ein gesellschaftliches Naturrecht entgegen (1941: S. 136-182) und plädiert Fraenkel für eine "Einheitsfront aller Naturrechtler“ (1941: S. 17) aus Demokraten, Katholiken, Sozialisten und Marxisten gegen Hitler. '

${ }^{65}$ Fraenkel 1951, S. 31.

66 Vgl. Fraenkel 1958c. 
Naturrecht, der Vernunft im rationalistischen Naturrecht, der Tradition der konservativen Revolutionskritik eines Burke und dem Protest im sozialistischen Naturrecht. Differenzen zwischen den vier Ansätzen bezeichnet Fraenkel als „Familienstreitigkeiten“ ${ }^{67}$ In der entscheidenden Frage seien sich alle vier Familienangehörigen einig, daß es den Staat in Schranken weisende überpositive Rechtsgrundsätze zugunsten von Individualrechten gebe. Angesichts der bekannten Existenz vieler Naturrechtslehren mit antidemokratischer oder rassistischer Stoßrichtung bleiben Fraenkels Überlegungen jedoch ausgesprochen unbefriedigend.

Das gleiche gilt für den epistemischen Status naturrechtlicher Aussagen. In einer unveröffentlichten Argumentationsskizze von 1961 aus seinem Nachlaß versucht er, das Naturrecht auf folgende Weise zu plausibilisieren:

\begin{abstract}
„Es lassen sich für den Rechtspositivismus zahlreiche logische Argumente anführen, die nur schwer zu widerlegen sind. Und doch leidet der Rechtspositivismus an einem Mangel. Wenn die Anwendung der Gesetze nicht nur in Ausnahmefällen, sondern immer wieder und wieder mit dem in Widerspruch gerät, was unser Gewissen, unser instinktives Rechtsgefühl als gerecht und billig ansieht, dann [...] tritt ein Spannungsverhältnis zwischen dem Gesetzgeber und dem Gesetzesunterworfenen in Erscheinung, das sich auf die Dauer als untragbar erweist [...] Der Kampf zwischen positivem Recht und Naturrecht ist ein Menschheitsproblem, das wohl stets eine ungelöste Aufgabe bleiben wird. Die starren Rechtspositivisten, die dem Naturrecht jegliche Bedeutung absprechen, und die unkritischen Naturrechtler, die glauben, das positive Recht mit einer großen Handbewegung beiseite schieben zu können, machen es sich beide Male zu leicht" ${ }^{68}$
\end{abstract}

Da die Wertebegründung in Fraenkels Verständnis ein essentieller Bestandteil von Politikwissenschaft ist, irritiert es umso mehr, daß er der von ihm selbst angeschnittenen Begründungsfrage immer wieder auszuweichen versucht. Mehrfach findet sich bei ihm ein funktionales Argument für naturrechtliche Normen. Es lautet, daß Demokratien ohne den Glauben seiner Bürger an naturrechtliche Normen nicht lange bestehen können. In diesem Zusammenhang führt Fraenkel die USA als besonders positives Beispiel an. ${ }^{69}$ Warum diese Werte aber eine normative Anerkennungswürdigkeit verdienen, wird von ihm - bei aller Kritik am Verlust des Vertrauens in die Wirksamkeit des naturrechtlichen Moments in der Politik etwa bei Carl Schmitt ${ }^{70}$ nicht ausführlicher thematisiert. Selbst wenn Fraenkel - wie in einem seiner letzten Aufsätze - hervorhebt, wie „fundamental wichtig" es sei, sich mit „dem Problem der Geltung" naturrechtlicher Normen auseinanderzusetzen, geht es ihm doch wieder nur darum, auf die „Wirksamkeit“"71 dieser Normen hinzuweisen.

Ich vermute, daß Fraenkel einen Ansatz bei der Begründung naturrechtlicher Normen verfolgt, der auf (im angenommenen Normalfall) tiefsitzende Intuitionen setzt. Den meisten Vertretern seiner Generation wirft er ein schlichtes Selbstmißverständnis vor. Sie besäßen ,weder die innere Kraft [...], sich offen zu der Geltung eines naturrechtlich

${ }^{67}$ Fraenkel 1958c, S. 165.

${ }^{68}$ Fraenkel 1961, S. 3 f.

${ }^{69}$ Vgl. Fraenkel 1960c, S. 28, 104, 345 f.

${ }^{70}$ Vgl. ebd., S. 346.

${ }^{71}$ Alle Zitate Fraenkel 1969, S. 355. 
legitimierten Wertsystems zu bekennen, noch den Mut aufbring(en), dessen Geltung für die Politik zu leugnen“. ${ }^{72}$ Erst wenn es wirklich um das Ganze geht, ,entdecken ${ }^{6}$ die intellektuellen Kritiker des Naturrechts ihre Intuitionen und verteidigen es. Fraenkels Überlegungen im Doppelstaat beschreiben wohl auch seine Nachkriegsposition zutreffend: „Seit mehr als 100 Jahren ist die Naturrechtslehre in Mißkredit geraten. Stets und von neuem ist sie von der Staats- und Politikwissenschaft (von der Rechtsphilosophie ganz zu schweigen) widerlegt worden. Und doch hat sie ihre Lebenskraft nicht eingebüßt. Seit Generationen gibt es einen Menschentyp, der jede Art von Naturrecht mit seinem Intellekt verneint und der sich gleichzeitig zu dessen Anerkennung durch sein Gewissen verpflichtet erachtet" ${ }^{73}$ In einer Formulierung aus dem Jahre 1970 klingt diese Sichtweise noch nach, wenn er behauptet, daß das Naturrecht, selbst wenn es mit „einer Heugabel herausgeworfen wird, doch stets wiederkehrt" ${ }^{74}$

Daß Fraenkels Überlegungen zum Thema Naturrecht nicht den stärksten Teil seiner Politischen Theorie ausmachen, ist ihm selbst nicht verborgen geblieben. In einem Brief kurz vor seinem Tod räumte er ein, daß er das Geltungsproblem des Naturrechts nicht befriedigend gelöst habe und ihm als Geltungsinstanz nur das Gewissen einfalle. ${ }^{75}$ In diese normative Lücke sind in der Fraenkel-Nachfolge drei unterschiedliche Lösungsstrategien gesprungen.

- Hans Kremendahl und zwischenzeitlich Winfried Steffani suchten einen Ausweg, in dem sie auf die naturrechtlichen Thesen Fraenkels verzichteten. Kremendahl plädierte statt dessen für den Anschluß der Pluralismustheorie an den Kritischen Rationalismus. $^{76}$

- Alexander Schwan und Joachim Detjen zogen eine Konsequenz, die in die andere Richtung zielt. Beide reklamierten, daß ein zu Ende gedachter Fraenkel den normativen Schulterschluß mit neueren Naturrechtslehren suchen müsse. ${ }^{77}$

- Drittens schließlich machte Peter Massing den Vorschlag, die normative Lücke bei Fraenkel durch Versatzstücke aus der Habermasschen Diskursethik zu füllen. ${ }^{78}$

Fassen wir zusammen: Das Naturrecht ist von zentraler Bedeutung für Fraenkels Gemeinwohlkonzeption. Leider finden wir bei Fraenkel weder eine klar umgrenzte Beschreibung dessen, was die naturrechtlichen Werte umfassen noch eine überzeugende Bestimmung des Status naturrechtlicher Normen. Fraenkels engagiertes Insistieren auf dem Naturrecht kann diese Schwächen nicht verdecken.

\footnotetext{
${ }^{72}$ Fraenkel 1963b, S. 342.

${ }^{73}$ Fraenkel 1941, S. 141.

${ }^{74}$ Fraenkel 1970, S. 17.

75 Vgl. Kremendahl 1977, S. 60.

76 Vgl. Kremendahl 1977, Steffani 1980.

77 Vgl. Schwan 1978, Detjen 1988.

${ }^{78} \mathrm{Vgl}$. Massing 1979.
} 


\section{Schlußbemerkung: Fraenkels Konzept und die drei politikwissenschaftlichen Modelle des ,Gemeinwohls ${ }^{6}$}

Eingangs wurden einige Bedenken genannt, auf die eine heutige Neu-Thematisierung von ,Gemeinwohl' stößt. Inwieweit ist auch Emst Fraenkels Gemeinwohlkonzeption anfällig für diese Einwände?

- Ein Bedenken lautet, daß dem Gemeinwohl ein ideologischer Gebrauch anhafte. Auch Fraenkel war sich dieser Problematik bewußt. Er bemühte sich, ihr in seiner Gemeinwohlkonzeption dadurch gerecht zu werden, daß er die Offenheit und Kommunikationsorientiertheit des politischen Prozesses in der pluralistischen Demokratie als Schutzvorrichtungen gegen einen ideologischen Gebrauch der Gemeinwohlformel konzipiert.

- Ein weiterer Einwand ist, daß das Konzept des Gemeinwohls für traditionelle, nicht aber für moderne und ausdifferenzierte Gesellschaften tauge. Auch in diesem Punkt kann Fraenkel nicht nur ein Problembewußtsein attestiert werden, sondern hat seine Konzeption den Bezug auf moderne ausdifferenzierte Gesellschaften geradezu zur Voraussetzung.

- Ein weiterer Einwand zielt schließlich auf die Unschärfe der vielstimmigen Rede vom Gemeinwohl, da sie weder das Wer? ihrer Bezugsgröße, noch das Was? ihres Inhalt, auch nicht des Wann? Ihres Zeithorizonts geschweige denn das Wie? ihre Entstehung und Realisierung eindeutig angebe. Fraenkels Antworten auf diese Fragen fallen unterschiedlich ausführlich und nicht gleichermaßen überzeugend aus. Am ausführlichsten geht er auf die Frage des,Wie?' in seinen Darlegungen der vielfältigen prozeduralen Aspekte der politischen Willensbildung in einer pluralistischen Demokratie ein. Die konkreten Ergebnisse des politischen Prozesses sind bereits auch ein wesentlicher Teil der Antwort auf die Frage des ,Was?'; der andere Teil besteht in den Überlegungen Fraenkels zum Themenkomplex Naturrecht. Am wenigsten reflektiert Fraenkel die Frage des ,Wer? ${ }^{\star}$, zu denen er der damaligen Konvention folgend die jeweiligen Angehörigen eines politischen Gemeinwesens rechnet.

Insgesamt fällt die von Fraenkel so anschaulich in Szene gesetzte Antwort auf die ,Gretchenfrage 6 der Politikwissenschaft, wie sie es wohl mit dem Gemeinwohl halte, mindestens ebenso gewunden und mehrdeutig wie die Antwort des Doktor Faust in Goethes Schauspiel aus.

- Zum einen ist Fraenkels Terminologie des ,Gemeinwohl a priori' und ,Gemeinwohl a posteriori` mißverständlich. Bei näherer Betrachtung stellte sich heraus, daß sich auch das Gemeinwohl a posteriori im Rahmen a priori geltender Normen befinden muß. Aus Fraenkels (erster und) materialer Kritik an Rousseau geht hervor, daß ihm eine Bezugnahme auf das empirische a posteriori politischer Prozesse nicht genügt. Um die Resultate politischer Prozesse inhaltlich bewerten zu können, bedarf es Werte, die - so scheint Fraenkel es zu meinen - außerhalb der Dispositionsgewalt der Menschen stehen. Diese Naturrechtsprinzipien werden (in Fraenkels Sprache) als Heteronomie vorgefunden und nicht autonom im politischen Prozeß erzeugt. Auch die westliche Demokratie verfügt somit über ein Moment des Heteronomen; eine Eigen- 
schaft, mit der Fraenkel ansonsten totalitäre Gemeinwohlvorstellungen charakterisiert.

- Zum zweiten bleibt der inhaltliche Kern des Gemeinwohls bei Fraenkel unklar. Welch Werte sind es, denen Fraenkel naturrechtlichen Status zuweist? Auch wenn er sie als abstrakt bezeichnet, erhebt Fraenkel den Anspruch, daß sie doch konkret genug sein sollen, um Politikwissenschaftler und Bürger zu befähigen, Ergebnisse politischer Prozesse auf ihren Gemeinwohlgehalt zu prüfen. Die beiden von ihm angeführten Grundwerte sind Gerechtigkeit und Billigkeit. Die ,Billigkeit' ${ }^{6}$ wird nicht näher ausgeführt und nur wenig besser steht es um den Inhalt von ,Gerechtigkeit‘. Erkennbar wird lediglich, daß Fraenkel neben Menschenwürde, Freiheit und Gleichheit eine distributive Auslegung der Gerechtigkeit vorschwebt. - Im Kontext der Überlegungen der Arbeitsgruppe ,Gemeinwohl und Gemeinsinn" der BerlinBrandenburgischen Akademie der Wissenschaften hat diese Festlegung zumindest polemischen Wert. Karsten Fischer und Herfried Münkler (1999) haben in dem bereits eingangs zitierten Aufsatz, der die Vorüberlegungen zu der Arbeitsgruppe resümiert, den Gemeinwohlbegriff auf die partikularistische und den neuzeitlichen Gerechtigkeitsbegriff auf die universalistische Seite der Moralphilosophie geschlagen. Die von Fraenkel als normativen Kern des Gemeinwohls vorgetragenen Elemente weisen dagegen eher in die universalistische Richtung. Fraenkel spricht in einem geradezu pathetischen Sinne von Gerechtigkeit und reklamiert dafür einen naturrechtlichen und damit universellen Status. So wackelig Fraenkels eigener Begründungsversuch ist; aus den Überlegungen Fraenkels folgt die These, daß die Gemeinwohlfrage sinnvoll nur im Rahmen einer Theorie der Gerechtigkeit diskutiert werden kann. Auch für die Polemik der beiden Autoren gegen die „distributive Auslegung “79 von Gerechtigkeit bietet Fraenkel wenig Schützenhilfe. Normativ wie funktional sah er in der Herstellung einer „gerechten Sozialordnung“ ein, wenn nicht das zentrale Moment moderner Demokratien. Der „Fortbestand der westlichen Demokratien" so Fraenkel, „hängt maßgeblich davon ab, daß das sozialökonomische Substrat der Verfassungs- und Rechtsordnung nicht in flagranten Widerspruch zu den Wertvorstellungen gerät, die das politische Denken erheblicher Gruppen der Bevölkerung bestimmen". 80

- Am wenigsten überzeugt schließlich die philosophische Seite in Fraenkels Gemeinwohlkonzeption. Zwar spricht er sich gegen wissenssoziologische und ideologiekritische Dekonstruktionen naturrechtlicher Vorstellungen aus; zugleich gibt er aber immer wieder $\mathrm{zu}$ erkennen, $\mathrm{da} ß$ er vom historischen Index in naturrechtlichen Vorstellungen weiß. Am ehesten überzeugt noch seine an Intuitionen appellierende These, daß die meisten klugen Kritiker des Naturrechts Opfer eines Selbstmißverständnisses seien und erst in solchen Situationen zu Klarheit über ihre Zustimmung zum Naturrecht gewinnen, wenn sie persönlich mit Terror und Unmenschlichkeit konfrontiert werden.

\footnotetext{
79 Münkler/Fischer 1999, S. 241.

${ }^{80}$ Fraenkel 1963b, S. 351.
} 
Worin bestehen heute noch aktuelle Momente der Fraenkelschen Konzeption für eine Renaissance des ,Gemeinwohls' in der Politischen Theorie? Um diese Frage zu beantworten, ist ein erneuter Blick auf die eingangs vorgestellten drei Gemeinwohlmodelle hilfreich, dem objektivistischen, dem Schnittmengen- und dem deliberativen Modell.

(a) Die Grundannahme des objektivistischen Modells besteht in der Vorstellung eines ,objektiven' Gemeinwohls, anhand dessen das Gemeinwohl sowohl erkennbar sein soll als auch als Orientierungspunkt praktischer Politik fungieren können soll. Fraenkel Kritik an diesem Modell ist schlagend: in seiner Terminologie handelt es sich hierbei um ein Gemeinwohl a priori, das letztlich zu einem totalitären politischen System führe.

(b) Das Schnittmengenmodell wurde als Interessenallgemeinheit des generalisierten Egoismus beschrieben. In diesem Modell besteht das Gemeinwohl aus der Schnittmenge aller empirisch vorfindlichen Interessenlagen mit der institutionellen Konsequenz einer möglichst breit gestreuten Veto-Positionierung politischer Akteure bei dem nur solche Inhalte das Gütesiegel ,Gemeinwohl' reklamieren können, die von allen Angehörigen eines politischen Systems geteilt werden.

(c) Das deliberative Modell als dritte Vorstellung von Gemeinwohl zielt auf eine kommunikative Allgemeinheit, bei dem die Genese des Gemeinwohls zwar von gegebenen Interessen und Präferenzen ausgeht, diese aber in einen Prozeß der argumentativen Auseinandersetzung überführt werden, so daß am Ende ein Ergebnis stehen kann, das für alle Betroffenen gleichermaßen akzeptabel ist.

Aus demokratietheoretischer Sicht besteht der Reiz des deliberativen Modells darin, daß es die eingangs genannten Unschärfen des Gemeinwohlbegriffs in der Weise konzeptionell aufnehmen kann, daß es deren Beantwortung zum Gegenstand praktischer Debatten über die Bestimmung des Gemeinwohls selbst macht. Auf diese Weise rückt die Beantwortung der eingangs formulierten vier offenen Fragen - nämlich wer von dem ,Gemein' umfaßt werden soll und wer nicht (also die soziale Dimension), was unter deren ,Wohl' zu verstehen ist und wie es festzustellen ist (die sachliche Dimension), welcher Zeithorizont Gegenstand der Debatte ist (die temporale Dimension) und schließlich der Frage, wie ein auch immer bestimmtes Gemeinwohl politisch zu realisieren ist (die praktische Dimension) - von der Ebene der akademischen Kritik auf die Ebene konkreter politischer Institutionalisierungsmodalitäten rückt. Die Aufgabe politischer Institutionen im Rahmen des deliberativen Gemeinwohlmodells ist es, die Beantwortung der vier genannten Fragen jedes Mal zu fordern und damit die politischmoralische Reflektion der politischen Akteure zu fördern. Institutionen werden damit zu Orten der Gemeinwohlkompetenz. Zwar ist die sachlich und moralisch adäquate Beantwortung dieser Fragen dadurch natürlich in keiner Weise garantiert, mit einer klugen Institutionalisierung kann das deliberative Gemeinwohlkonzept für sich aber wenigstens in Anspruch nehmen, die sozio-moralischen Ressourcen der Bürger optimal auszuschöpfen. Bei der Frage nach möglichen institutionellen Reformen zur Förderung des Gemeinwohls treffen sich dann auch die bundesdeutschen politikwissenschaftlichen Diskurse über ,Gemeinwohl', ,Tugenden' und ,Bürgergesellschaft ${ }^{6}$ mit der Habermasschen Version des Prozeduralismus und dem Gemeinwohlkonzept des, highest common 
concern von Robert E. Goodin. ${ }^{81}$ Mit Bezug auf die genannten vier Dimensionen würde dies bedeuten, politische Institutionen danach auszurichten,

- daß sie in der sozialen Dimension zunächst universalistisch orientiert sind und die Begründungslast zuungunsten von Ausschlußregeln festlegen,

- daß sie in der sachlichen Dimension ihre Beteiligungsformen und Verfahren an den kognitiven Zumutungen politischer Entscheidungen in komplexen Gesellschaften orientieren;

- daß sie in der temporalen Dimension die Gegenwart nicht zu ungunsten der Zukunft diskontieren,

- und daß sie in der praktischen Dimension die nicht-intendierten Konsequenzen politischer Entscheidungen zu antizipieren vermögen.

Dieses Anforderungsprofil ist nicht nur hoch, sondern es impliziert strenggenommen auch konkurrierende Logiken bei der institutionellen Beantwortung der vier Teilfragen. Erforderlich ist ein kluger institutioneller Mix, bei dem sich der Parlamentarismus den Formen unmittelbarer Partizipation als überlegen erweist. ${ }^{82}$

Die Fraenkelsche Gemeinwohlkonzeption weist Anknüpfungspunkte sowohl zu dem Schnittmengen- wie zu dem deliberativen Modell auf. Die genaue Verortung seiner Überlegungen zwischen diesen beiden Modellen hängt ganz wesentlich davon ab, wie weit sich die von Fraenkel in einigen Arbeiten ${ }^{83}$ stärker partizipativ akzentuierten prozeduralen Grundnormen, die auf eine kommunikative Öffnung politischer Willensbildungsprozesse zielen, interpretativ herausheben lassen. Werden die prozeduralen Grundnormen in Fraenkels Demokratietheorie als politische Kommunikationsrechte mit einem normativen Eigensinn versehen, dann ergeben sich durchaus Anknüpfungspunkte an die heutige Debatte über deliberative Demokratie. Doch um diese und in Verbindung damit ein politikwissenschaftliches Gemeinwohlkonzept weiterzuentwickeln, bieten Fraenkels Überlegungen wenig systematisch weiterführende Hinweise. Die Probleme und Fragestellungen der gegenwärtigen Gemeinwohldebatte lassen sich in diesem Fall nicht durch den Blick in die politikwissenschaftliche Ideengeschichte lösen.

\section{Literaturverzeichnis}

Buchstein, H. (1998), Ernst Fraenkel als Klassiker?, in: Leviathan 26, S. 458-481.

Buchstein, H. (2000), Von Max Adler zu Ernst Fraenkel. Demokratie und pluralistische Gesellschaft in der sozialistischen Demokratietheorie der Weimarer Republik, in: Demokratisches Denken in der Weimarer Republik, hg. v. Christoph Gusy, Baden-Baden, S. 534-607.

Buchstein, H. (2001), Bürgergesellschaft und Bürgerkompetenz. Erscheint, in: Bürgergesellschaft und Zivilgesellschaft, hg. v. G. Breit u. P. Massing, Schwalbach/Ts., S. 8-18.

Buchstein, H./Göhler, G. (Hg., 2000), Vom Sozialismus zum Pluralismus. Beiträge zu Leben und Werk von Ernst Fraenkel, Baden-Baden .

\footnotetext{
${ }^{81}$ Vgl. Münkler 1992, Habermas 1996, Goodin 1996, Buchstein 2001, sowie als Versuch, die Verbindung zwischen diesen Debatten herzustellen, Schmalz-Bruns 1995.

${ }^{82}$ Vgl. Lepsius 1999.

${ }^{83}$ Insbesondere Fraenkel 1960a, 44 f.; 1964a, S. 72 f., 1966a, S. 147 f.
} 
Dahl, R. A. (1963) A Preface to Democratic Theory, Chicago.

Detjen, J. (1988), Neopluralismus und Naturrecht, Paderborn u. a.

Eisfeld, R. (1972), Pluralismus zwischen Liberalismus und Sozialismus, Stuttgart.

Fraenkel, E. (1927), Zur Soziologie der Klassenjustiz, in: Ders. 1999, S. 177-211.

Fraenkel, E. (1928), Staat und Gewerkschaften, in: Ders. 1999, S. 285-292.

Fraenkel, E. (1929), Zum Verfassungstag, in: Ders. 1999, S. 358-364.

Fraenkel, E. (1932a), Um die Verfassung, in: Ders. 1999, S. 496-509.

Fraenkel, E. (1932b), Chronik Mai/Juni 1932, in: Ders. 1999, S. 579-584.

Fraenkel, E. (1932c), Chronik Juli/August 1932, in: Ders. 1999, S. 585-591.

Fraenkel, E. (1984), Der Doppelstaat [1941], Frankfurt/M.

Fraenkel, E. (1951), Korea - Ein Wendepunkt im Völkerrecht?, Berlin.

Fraenkel, E. (1958a), Die repräsentative und plebiszitäre Komponente im demokratischen Verfassungsstaat, in: Ders. 1991, S. 153-203.

Fraenkel, E. (1958b), Parlament und öffentliche Meinung, in: Ders. 1991, S. 204-231.

Fraenkel, E. (1958c), Staat und Einzelpersönlichkeit, in: Vorträge, gehalten anläßlich der Hessischen Hochschulwochen für staatswissenschaftliche Fortbildung, Bd. 20, Bad Homburg, S. 150-166.

Fraenkel, E. (1960a), Historische Vorbelastungen des deutschen Parlamentarismus, in: Ders. 1991, S. 23-46.

Fraenkel, E. (1960b), Deutschland und die westlichen Demokratien, in: Ders. 1991, S. 48-67.

Fraenkel, E. (1960c), Das amerikanische Regierungssystem, Köln u. Opladen.

Fraenkel, E. (1961), Naturrecht und Rechtspositivismus, Unveröffentlichtes Manuskript, 5 Seiten, Nachlaß E. Fraenkel, Bundesarchiv Koblenz, BAK 1274, Bd. 8.

Fraenkel, E. (1963a), Demokratie und öffentliche Meinung, in: Ders. 1991, S. 232-260.

Fraenkel, E. (1963b), Die Wissenschaft von der Politik und die Gesellschaft, in: Ders. 1973, S. 337-353.

Fraenkel, E. (1964a), Strukturdefekte der Demokratie und deren Überwindung, in: Ders. 1991, S. 68-94.

Fraenkel, E. (1964b), Der Pluralismus als Strukturelement der freiheitlich-rechtsstaatlichen Demokratie, in: Ders. 1991, S. 297-325.

Fraenkel, E. (1966a), Ursprung und politische Bedeutung der Parlamentsverdrossenheit, in: Ders. 1991, S. 137-150.

Fraenkel, E. (1966b), Möglichkeiten und Grenzen politischer Mitarbeit der Bürger in einer modernen parlamentarischen Demokratie, in: Ders. 1991, S. 261-276.

Fraenkel, E. (1967a), Die ordnungspolitische Bedeutung der Verbände im demokratischen Rechtsstaat, in: Ders. 1991, S. 277-296.

Fraenkel, E. (1967b), Universitas litterarum und pluralistische Demokratie, in: Ders. 1973, S. 354-368.

Fraenkel, E. (1969), Strukturanalyse der modernen Demokratie, in: Ders. 1991, S. 326-358.

Fraenkel, E. (1970), Der Streit um die Anerkennung der DDR im Licht der politischen Wissenschaft, in: Aus Politik und Zeitgeschichte B 17/70, S. 3-17.

Fraenkel, E. (1973), Reformismus und Pluralismus, Hamburg.

Fraenkel, E. (1991), Deutschland und die westlichen Demokratien, erw. Neuausgabe, hg. v. A. v. Brünneck, Frankfurt/M.

Fraenkel, E. (1999), Gesammelte Schriften Bd. 1, Recht und Politik in der Weimarer Republik, hg. v. H. Buchstein u. R. Kühn, Baden-Baden.

Goodin, R. E. (1996), Institutionalizing the Public Interest, in: American Political Science Review 90, S. 331-343.

Habermas, J. (1996), Drei normative Modelle der Demokratie, in: Ders., Die Einbeziehung des Anderen, Frankfurt/M., S. 277-292.

Kremendahl, H. (1977), Pluralismustheorie in Deutschland, Leverkusen.

Lepsius, O. (1999), Die erkenntistheoretische Notwendigkeit des Parlamentarismus, in: Demokratie und Freiheit, hg. v. M. Bertschi u. a., Stuttgart u. a., S. 123-180. 
Massing, P. (1979), Interesse und Konsensus, Opladen.

Mills, C. W. (1981), The Power Elite [1956], New York.

Münkler, H. (1992), Politische Tugend, in: Ders. (Hg.), Die Chancen der Freiheit, München, S. 25-46.

Münkler, H./Fischer, K. (1999), Gemeinwohl und Gemeinsinn. Thematisierung und Verbrauch soziomoralischer Ressourcen in der modernen Gesellschaft, in: Berlin-Brandenburgische Akademie der Wissenschaften. Berichte und Abhandlungen, Bd. 7, Berlin, S. 237-265.

Narr, W.-D. (1969), Pluralistische Gesellschaft, Hannover.

Offe, C. (1972), Strukturprobleme des kapitalistischen Staates, Frankfurt/M.

Offe, C. (2002), Wessen Wohl ist das Gemeinwohl? In: Gemeinwohl und Gemeinsinn. Rhetoriken und Perspektiven sozial-moralischer Orientierung, hg. v. H. Münkler u. K. Fischer, Berlin, S. 55-76.

Scharpf, F. W. (1991), Die Handlungsfähigkeit des Staates am Ende des 20. Jahrhunderts, in: Politische Vierteljahresschrift 32, S. 621-634.

Schmalz-Bruns, R. (1995), Reflexive Demokratie. Die demokratische Transformation moderner Politik, Baden-Baden.

Schmalz-Bruns, R. (1996), Gemeinwohl. Notizen zu einem Forschungsprogramm. Unveröffentlichtes Manuskript, 7 Seiten.

Schubert, G. (1960), The Public Interest, Glencoe.

Schwan, A. (1978), Grundwerte der Demokratie. Orientierungsversuche im Pluralismus, München.

Steffani, W. (1980), Pluralistische Demokratie, Opladen.

Talmon, J. L. (1952), The Origins of Totalitarian Democracy, London. 